\title{
Philonsorbonne
}

8 | 2014

Année 2013-2014

\section{Le voile des femmes arabes, point de division entre espace public et religiosité}

\section{Diana TASINI}

\section{(2) OpenEdition \\ Journals}

Édition électronique

URL : https://journals.openedition.org/philonsorbonne/619

DOI : 10.4000/philonsorbonne.619

ISSN : $2270-7336$

Éditeur

Publications de la Sorbonne

\section{Édition imprimée}

Date de publication : 1 janvier 2014

Pagination : 189-199

ISSN : 1255-183X

\section{Référence électronique}

Diana TASINI, «Le voile des femmes arabes, point de division entre espace public et religiosité »,

Philonsorbonne [En ligne], 8 | 2014, mis en ligne le 19 janvier 2014, consulté le 08 juin 2021. URL:

http://journals.openedition.org/philonsorbonne/619; DOI : https://doi.org/10.4000/philonsorbonne. 619

(c) Tous droits réservés 


\title{
Le voile des femmes arabes, point de division entre espace public et religiosité
}

\author{
Diana TASINI
}

Mon propos, bien que non exhaustif vu l'ampleur du sujet, se veut une réflexion sur le port du voile islamique, souvent perçu comme une menace de l'espace public en dehors du monde arabe, et sur le fait qu'il représente, dans ses dimensions sociale et privée, plus que beaucoup d'autres symboles religieux, un emblème prismatique de la sécurité collective comme des droits des femmes. Une double revendication se dessine dans le débat politique, sociologique ou juridique actuel: celle de la laïcité de l'État et de l'appartenance à une collectivité, qui revendique une place grandissante dans le monde occidental, sans avoir à renoncer à ses traditions culturelles et religieuses. La frontière juridique de la tolérabilité/intolérabilité envers le voile intégral porté dans l'espace public est en évolution constante et fluctue selon l'optique dans laquelle l'on se place : sécurité publique, égalité entre les hommes et les femmes, dignité de la femme, liberté de culte et d'expression religieuse, etc. En modifiant le point de vue, le cadre de référence législatif et de valeur change, ce qui se traduit par des différences de règles et de comportements.

La modernité européenne, telle qu'elle s'est affirmée au $\mathrm{XX}^{\mathrm{e}}$ siècle, est décrite essentiellement comme un produit de la séparation entre l'Église et l'État : les pays européens ont construit leur idée de modernité en reléguant la religion et le sacré dans la sphère privée. L'exclusion de la religion, au titre de pierre angulaire des relations publiques, a permis de garantir les droits des individus, notamment des femmes. Selon cette lecture de l'Europe moderne, les musulmans représentent un danger de par leurs pratiques religieuses publiques et affichées, tel que le port du voile pour les femmes, et envahissent l'espace public, actuellement territoire dominant de l'État laïc. Malgré l'absence d'expression au niveau institutionnel, la revendication croissante de visibilité de l'islam, en Europe ou ailleurs, non exempte d'influences politiques parfois révolutionnaires, ne peut passer inaperçue. 
Dans cette optique, le voile des femmes musulmanes ne se limite pas à un simple symbole d'inégalité des sexes imposé par un mécanisme de contrôle masculin sur le corps de la femme, concept inacceptable pour des pays fondés sur le principe d'égalité des sexes, mais un danger pour la laïcité (conçue comme un rôle de l'État dans la défense des individus contre les ingérences religieuses) et pour les conquêtes issues de la séparation entre l'Église et l'État. Le voile est, par conséquent, perçu comme un refus plus général des valeurs européennes.

Victime fréquente d'un membre de sa famille, la femme voilée en est également la complice. Dans le débat européen, l'on constate que la vision de la femme oscille constamment entre celle de créature opprimée et de provocatrice contre l'ordre constitué, piège tendu à la sécularisation. L'islam avec ses symboles peut, par conséquent, représenter une sorte de subversion qui fait naître le besoin, chez nombre de personnes, de réguler sa diffusion dans un espace public, par une réglementation prévue à cet effet; l'exhibition de son credo religieux placé avant toute chose, même les valeurs dominantes de la culture d'accueil, du domaine privé au domaine public, du niveau personnel au collectif, entre souvent en conflit avec les valeurs occidentales et laïques de la modernité. Les personnes qui adoptent une forme radicale de présentation de leur identité religieuse et culturelle se trouvent souvent en désaccord avec les règles nationales et laïques régissant l'espace public, et revendiquent ouvertement leurs us et coutumes ainsi que leur identité religieuse.

La visibilité claire des symboles et des pratiques religieuses est révélatrice de la mutation qui s'opère au sein de la laïcité sociale : porter le voile ou la barbe, revendiquer le droit à disposer d'endroits et d'heures pour la prière, même sur le lieu de travail, ou suivre des régimes alimentaires spécifiques, signifient à la fois se distinguer immédiatement et se faire respecter dans l'espace public.

Pour de nombreuses femmes immigrées, la religion est vécue non seulement comme une question personnelle, mais aussi comme une richesse à partager en communauté : la sociologue Peggy Levitt souligne que les fils d'immigrés, en quête d'un outil pour s'insérer dans la dimension publique et politique de leurs pays de résidence, se tournent vers la religion (quelle qu'elle soit) puisqu'elle leur fournit un cadre de référence pour l'orientation de leur propre identité.

Comme l'affirme Sumaya Abdel Qader, écrivain musulman italien, l'islam ne peut plus être conçu comme un phénomène extérieur au monde occidental : les pratiques musulmanes augmentent au quotidien dans la vie publique et l'incidence dans l'imaginaire social fait désormais partie intégrante de la société européenne, qui se transforme rapidement et compte également des jeunes et des femmes voilées.

L'un des symboles les plus célèbres et les plus éclatants est, précisément, le voile partiel ou intégral des femmes. En réalité, même si le voile fait partie des stéréotypes occidentaux sur la civilisation arabo- 
islamique et suggère des atmosphères des Mille et une nuits, le besoin de masquer le corps de la femme (le plus souvent), mais aussi de l'homme, était déjà répandu dans toutes les cultures méditerranéennes avant l'islamisation, de même que dans d'autres contextes, à l'instar des traditions populaires du Sud de l'Italie.

Puisque l'emploi de termes arabes pourrait donner lieu à des doutes d'interprétation, surtout lorsque les mots peuvent s'employer dans des sens légèrement différents en fonction $\mathrm{du}$ lieu et du contexte, une courte nomenclature de ces vêtements serait peut-être utile. Il est important de préciser qu'il existe plusieurs types de voile féminin dans les cultures islamiques. Ceux-ci sont liés pour la plupart aux différentes régions géographiques et vont de la couverture totale du corps (burqa, niqab) ou du simple dévoilement du visage (tchador, khimar), aux voiles légers à porter sur les vêtements habituels (abaya, jilbab) pour en arriver au voile minimal prévu par la charia (hijab). La confusion est fréquente entre ce qui relève de la tradition ou de la religion. En ce sens, le voile islamique est interprété, surtout par certains groupes, comme un symbole religieux plutôt que comme une tradition. En effet, une partie du débat doctrinaire religieux repose sur l'interprétation de sept versets du Coran dans lesquels apparaît le mot hijab, non de façon spécifique puisqu'il est fait allusion soit à un rideau permettant de se cacher pour prier, soit à une tente derrière laquelle se produit la révélation divine. Par ailleurs, deux autres passages du Coran contiennent des termes dérivant de la racine «kh-m-r» (« occulter quelque chose »), et le mot jalabib, dont la racine a pour signification d' «être revêtu de quelque chose » pour désigner le voile.

Avec le temps, le voile s'est mis à faire fonction de ligne de division entre l'espace public et la religiosité. Il en est ainsi dans deux pays européens, en l'occurrence la France et l'Italie. Ces dernières années, nulle part ailleurs comme en France, l'affaire du port du voile n'a autant divisé l'opinion publique, bien que l'on n'y recense que 4000000 de musulmans, la plupart d'origine nord-africaine, et que les problématiques ne concernent qu'une minorité d'entre eux. Pionnier en Europe dans la promulgation de lois en la matière, ce pays s'est en effet doté d'une législation avancée, tandis qu'en Italie le débat est plus que jamais ouvert et le processus législatif encore en cours.

Le 19 mars 2013, la Cour de cassation de Paris, dans l'arrêt $n^{\circ}$ 536, a réintégré à son poste de travail une salariée d'une école maternelle, qui avait été licenciée pour avoir porté le voile pendant son service, et ce en dépit des rappels à l'ordre garantissant le principe de laïcité. Cette décision a relancé le débat national sur l'ostentation des symboles religieux dans les lieux publics. La salariée avait bénéficié de l'appui de la Halde, autorité pour la lutte contre les discriminations, ce qui n'a pas empêché le Conseil des prud'hommes de confirmer son licenciement. 
Le débat sur le port du voile en France ne s'est pas arrêté aux polémiques législatives. Il a atteint un niveau plus théorique en entraînant une vaste discussion sur la sécularisation française, la fameuse laïcité de l'État, laquelle s'est muée au fil du temps d'idéal révolutionnaire (Déclaration de 1789) en pierre angulaire de la République (principes constitutionnels de 1946 et de 1958). La laïcité républicaine repose sur trois points fondamentaux : la liberté de choisir sa foi ou de s'abstenir de croire, l'égalité de tous les citoyens et la neutralité de l'État face à l'aspect religieux et à son influence. Ce concept rend l'expérience française unique au sein des constitutions occidentales modernes et démocratiques, et délimite la plate-forme de valeurs partagées qui unissent tous les membres de l'Étatcollectivité dans la dimension de la citoyenneté, abstraction faite de leur appartenance religieuse.

La conception de la laïcité propre à la sphère publique française reste toutefois un argument controversé : dès la fin de l'époque coloniale, une partie de l'opinion publique voulait bannir le voile (symbole d'assujettissement féminin et menace à la laïcité étatique), tandis qu'une autre partie a toujours défendu la liberté de le porter en dénonçant l'attitude néocolonialiste de la France.

En 2003, l'institution du Conseil français pour le culte musulman a modifié les relations politiques et publiques entre l'État français et l'islam. En effet, la naissance d'une organisation religieuse islamique est synonyme de reconnaissance publique de la présence musulmane en France, et par conséquent, l'acceptation politique de l'islam comme facteur indépendant du problème de l'immigration. Ce constat implique également l'existence d'un espace créé à l'initiative de l'État et destiné à la religion dans la sphère publique.

Quoi qu'il en soit, les premières concernées, à savoir les femmes, sont toujours restées au deuxième plan, hôtes indésirables d'une histoire dont elles étaient les actrices principales, jugées de façon trop hâtive comme les victimes plus ou moins conscientes d'une culture patriarcale et d'abus de l'autorité masculine. Entre elles aussi, les opinions liées, pour la plupart, à leurs origines ou à leurs traditions religieuses divergent.

De nombreuses musulmanes immigrées en Europe ont eu accès aux études supérieures, ont participé à la vie de la collectivité et à l'ordre du jour public sans être désormais contraintes de porter le voile. Elles l'ont fait de leur propre gré et ont transformé une vision de discrédit public potentiel en atout personnel. En vertu de ce libre choix, elles ont la sensation qu'un sens historique d'humiliation et de perte de dignité s'est mué en une recherche de distinction, de prestige et de pouvoir, parce qu'elles ne sont plus enfermées entre leurs quatre murs et que leur action est le fruit d'un libre choix. De nombreuses lettrées françaises favorables au port du voile soutiennent que les jeunes immigrées qui choisissent de porter le voile islamique, même en public, sont sous bien des aspects - comme l'émancipation, l'instruction, la culture juvénile, la conscience d'elles-mêmes et de leurs droits - plus 
proches des jeunes de leur âge que de leurs mères, génération antérieure constituée de femmes peu ou pas instruites et cloîtrées chez elles. Elles rectifient, parfois involontairement, l'image traditionnelle de la femme musulmane, souvent contrainte au port du voile par les membres de sa famille. Il est donc intéressant de remarquer que ces nouvelles musulmanes européennes détiennent un double capital culturel leur permettant de se mouvoir dans plusieurs secteurs publics ou privés : la maison, l'école, le travail, la politique, les associations juvéniles et les loisirs. D'une part, elles se définissent par leur religion, d'autre part, elles ont appris les techniques d'autoreprésentation dans les lieux publics et ont acquis un savoir universel laïc, ce qui suscite une perplexité difficile à masquer au sein de l'opinion publique.

Celles qui y sont opposées soulignent, en revanche, que le Coran ne fait allusion nulle part à l'obligation de se couvrir les cheveux et/ou le visage : selon elles, la Sura 24.31, fréquemment invoquée par les fondamentalistes, n'est qu'une invitation à la pudeur. Elles considèrent le voile intégral comme une mortification de la dignité féminine parce que la couverture totale du visage prive la femme de toute forme de socialisation: communiquer en public avec un interlocuteur dont l'identité est cachée est en effet très difficile du point de vue psychologique et sociologique, et peut créer des tensions et de la méfiance. La femme, dont l'identité est voilée et niée aux autres, est en quelque sorte isolée du monde extérieur. Elle s'assimile presque à une non-personne dans les relations élémentaires de la vie quotidienne et se voit priver de la libre manifestation de son individualité. Cet état de fait peut même donner lieu à des situations dangereuses, surtout pour la femme, puisque ses mouvements physiques sont entravés par ses vêtements. La burqa et le niqab ne constituent pas une simple barrière psychologique et physique avec le monde extérieur, signe annonciateur de méfiance, de désorientation et de discrimination à l'encontre de ces femmes, mais traînent dans leur sillage des problèmes d'intégration au sein d'une réalité européenne, où s'est affirmée une conception très différente de l'image et de la dignité féminines au fil du temps. En rappelant la thèse éclairée de Rousseau "la liberté consiste moins à faire sa volonté qu'à ne pas être soumis à celle d'autrui », l'autre problématique à prendre en considération est la coercition, bien souvent violente, qui contraint les femmes musulmanes à porter ce type de vêtement. Cette règle imposée par la famille, surtout par ses éléments masculins, repose sur la motivation religieuse justifiant le recours à des sanctions, éventuellement corporelles, à l'encontre des femmes qui refusent de se plier au joug familial. Les divergences à cet égard prennent parfois une tournure dramatique et dégénèrent: elles peuvent donner lieu à des actes aberrants, impossibles à ignorer parce qu'ils font trop de victimes.

Quel que soit le domaine, les législateurs français ont toujours accordé une grande attention aux droits des femmes en programmant sans cesse des interventions visant à atteindre l'égalité totale entre les hommes et les femmes, assorties d'initiatives destinées à favoriser la femme dans chaque 
secteur de la vie et des fonctions publiques avec des résultats probants. Toutefois, la France a été le seul pays à aller aussi loin sur la voie du prohibitionnisme et est devenue un symbole pour les autres nations qui souhaitent interdire le port du voile dans les lieux publics. À la différence du reste de l'Europe, où les propositions concernant ce genre d'interdiction sont présentées traditionnellement par les partis de droite et les mouvements nationalistes, en France le projet d'interdiction a été appuyé par une grande partie de la gauche (en provoquant d'importants clivages dans le mouvement féministe et parmi les intellectuels).

Un récapitulatif des principales étapes de l'histoire législative peut s'avérer utile :

- 1905 : promulgation de la loi qui établit la séparation entre les Églises et l'État, en particulier à l'encontre de l'Église catholique considérée alors comme la principale antagoniste de l'État républicain.

- 1989 : le Conseil d'État décrète que le port de symboles religieux n'est pas incompatible avec le principe de la laïcité de l'État, à moins que ces symboles n'aient pour objectif la propagande, le prosélytisme ou la provocation.

- 1994: proposition de loi visant à interdire tous les signes d'appartenance religieuse, présentée par un député du parti de centre droit.

- Au cours de la même année, le ministre de l'Éducation nationale publie une circulaire selon laquelle tous les symboles d'appartenance religieuse doivent être interdits dans les écoles. Seuls seront admis les signes discrets. Cette fois encore, le Conseil d'État intervient en rappelant les principes exprimés par le passé et en soulignant que la possibilité de trouver des solutions alternatives incombe, au cas par cas, aux enseignants et aux directeurs d'établissements.

- Juillet 2003 : le gouvernement charge une commission de préparer un document sur l'application du principe de laïcité dans l'espace public.

- Mars 2004: suite aux résultats de la commission, la loi $n^{\circ} 228 / 2004$ est promulguée à une très large majorité (par les députés de droite comme par les socialistes). Pour mettre en vigueur le principe de la laïcité de l'école publique, la loi interdit l'ostentation de symboles confessionnels (incluant non seulement le voile islamique, mais également la kippa juive et le turban sikh, en permettant pourtant de porter de petites étoiles de David, des mains de Fatima et des croix). L'adoption de cette loi a suscité de nombreux débats sur le respect de la liberté et de l'égalité de chaque citoyen, même si seules $14 \%$ des femmes musulmanes demeurant en France portaient le voile lors de sa promulgation.

- Année scolaire 2004/2005: entrée en vigueur de la loi 228. Pendant l'année, 44 étudiantes musulmanes et quatre étudiants sikhs 
sont expulsés des établissements scolaires. De nombreux étudiants choisissent en revanche la voie de l'éducation privée pour contourner l'interdiction. Pour ses opposants, la loi est la preuve que ce type de législation représente une discrimination à l'encontre de personnes que l'État, lui-même, prive du droit universel à l'éducation.

- Juin 2009 / janvier 2010 : une «mission d'information sur la pratique du port du voile intégral sur le territoire national » est instituée par le parlement français. Après six mois de travaux et d'auditions, la commission présente à l'Assemblée nationale un rapport qui qualifie la burqa de signe d'esclavage incompatible avec les principes de la République. Elle recommande la promulgation d'un acte législatif qui, eu égard à la nature particulière du service public, édicte des règles interdisant la burqa et le niqab dans les commerces et services publics. Elle se garde toutefois d'étendre cette interdiction à l'ensemble des lieux publics étant donné l'absence d'unanimité sur la question et le doute concernant la légitimité constitutionnelle d'une interdiction générale.

- Printemps 2011 : entrée en vigueur de la loi bipartite $n^{\circ} 1192$ du 11 octobre 2010, « interdisant la dissimulation du visage dans l'espace public ». La loi interdit, dans un espace public, le port d'un vêtement ou d'un accessoire qui dissimule le visage d'une personne, empêchant de la sorte sa reconnaissance ou son identification. L'article 2 précise que par «espace public» on entend les voies publiques et les lieux ouverts au public ou destinés à un service public. Certaines dérogations sont cependant prévues par des dispositions explicites (ex.: le port du casque pour les motocyclistes) en cas de nécessité de protection de l'anonymat (ex. : les témoins dans des procès particuliers), pour des raisons de santé (ex. : masques respiratoires ou cas d'épidémies), des motifs professionnels (ex.: forces de l'ordre, disciplines sportives particulières), ou encore en cas de fêtes populaires.

Comme on l'imagine aisément, le processus législatif n'a pas été simple. Des voix se sont élevées pour parler d'attentat à la dignité humaine, notamment de la femme, qui se trouve dans une situation d'humiliation insupportable en portant la burqa ou le niqab, ou de défense de l'ordre public et des principes fondateurs de l'État français, sans oublier du moins les questions posées par la commission parlementaire. En mars 2010, le Conseil d'État avait souligné l'inconstitutionnalité d'une interdiction qui ne serait pas limitée à des raisons de sûreté publique et à des lieux bien déterminés, de même que la difficulté de relier la dignité et l'égalité des sexes à des personnes qui choisissent délibérément de porter le voile intégral. D'autre part, la laïcité ne peut fournir le fondement à une limitation généralisée de l'expression de religiosité. Les juges suprêmes avaient donc exprimé de fortes réserves, non seulement sur la constitutionnalité de ce projet de loi, mais également sur sa compatibilité avec la Convention européenne pour la protection des droits de l'homme et des libertés fondamentales. 
Les doutes de constitutionnalité furent résolus par la décision du Conseil constitutionnel, qui précisa que le principe de liberté de l'individu peut être limité par le législateur lorsqu'il s'oppose aux intérêts publics. La nécessité de veiller à la sécurité, qui aurait été compromise par le fait de circuler dans les lieux publics avec le visage couvert, rend l'interdiction compatible avec la Constitution. Le Conseil mit cependant l'accent sur le besoin de réserve explicite dans l'application de la loi, en référence aux lieux de culte ouverts au public.

Bien que discutable, la loi française contre la burqa semble d'une certaine façon cohérente avec ce qui a été fait jusqu'à ce jour dans le pays et devrait permettre aux nombreuses jeunes femmes demeurant sur son territoire, du moins selon les défenseurs de sa promulgation, de choisir entre le port ou le refus du voile, au détriment des contraintes qui leur seraient imposées par leur propre famille. Selon les opposants à la loi, l'effet consistera en revanche à priver les femmes, qui voudraient opter pour le port du voile, de la liberté de se vêtir comme elles le jugent opportun, tandis que celles qui sont soumises aux pères ou aux maris autoritaires n'auront certes pas la force de se rebeller en dénonçant leur propre famille pour infraction à la loi et seront reléguées à une vie encore plus solitaire.

L'approche du thème du voile islamique en Italie peut permettre de saisir les différentes analogies. Au fil du temps, une partie de la doctrine italienne a souligné à quel point la délicatesse de la matière et l'hétérogénéité des situations (par exemple les cas de coercition ou d'ostentation spontanée, un degré d'intégration suffisant pour donner force de loi aux raisons d'une interdiction) qui naissent des sociétés multiculturelles auraient dû peser sur les solutions appliquées non seulement par la jurisprudence, mais également et surtout par le législateur. Quoi qu'il en soit, en Italie comme en France, la problématique soulevée par l'interdiction de la burqa est une question qui concerne un nombre non négligeable de personnes. Elle est toutefois fort pénalisante, principalement pour les générations d'immigrés ayant grandi dans leur pays d'origine et profondément liés à leurs traditions religieuses, alors que ces traditions ne font qu'un avec le droit dans les pays islamiques.

La Constitution italienne stipule que les relations entre l'État et les autres religions que la foi catholique sont régies par des interventions législatives. Ces dernières années, de nombreuses réformes organiques ont été approuvées sans parvenir pour autant à un consensus autour d'une loi sur la liberté de religion, et donc en maintenant le statu quo actuel.

Dans le cas de l'Italie, il est également utile de préciser certaines étapes marquantes :

- 1931 : l'article 85 du TULPS («texte unique de lois sur la sûreté publique »), approuvé par l'arrêté royal $\mathrm{n}^{\circ} 773$, interdit de circuler masqué dans un lieu public et prévoit, en cas de contravention à la loi, une sanction administrative. 
- 1975 : la loi 152, promulguée en guise de mesure contre le terrorisme, interdit le port de casques ou de vêtements susceptibles d'empêcher l'identification d'une personne dans un lieu public. Modifiée en 1977, la loi sera renforcée en 2005. Son interprétation est à l'origine de fortes divergences en jurisprudence: une première interprétation, basée sur l'article 19 de la Constitution stipulant le droit individuel de professer librement sa foi et d'en exercer le culte, même en public, si ce n'est dans les limites des bonnes mœurs, estime que la loi ne doit pas s'appliquer au cas du voile islamique en tant qu'expression de l'identité culturelle et religieuse constitutionnellement protégée. Comme l'a fait remarquer le chercheur Raffiotta (Université de Bologne) la loi «ne voit pas le jour pour régler le problème délicat et complexe du port de vêtements religieux, mais a été appliquée à des finalités et dans un contexte totalement différent de la société multiculturelle actuelle» (en l'occurrence les fameuses «années de plomb »). D'autre part, compte tenu des nécessités de protéger la sûreté publique, l'on estime que le voile islamique peut figurer au nombre des moyens aptes à entraver la reconnaissance de la personne.

- 1995 : le linistère de l'Intérieur publie la circulaire $n^{\circ} 4$ en suggérant aux administrations communales d'accepter les demandes de cartes d'identité assorties d'une photo montrant le sujet tête couverte «pour autant que les traits du visage soient bien visibles».

- 2000 : le ministère de l'Intérieur étend le principe de cette circulaire aux photos des permis de séjour en précisant que le tchador et le voile partiel, en tant que prescriptions religieuses, complètent la physionomie de qui les porte et contribuent à son identification. Toutefois, les officiers de l'état civil ou les fonctionnaires de police ont été nombreux à refuser les photos de femmes portant le voile, musulmanes ou sœurs catholiques, parce que la couverture de la tête ne permettait pas de déterminer la couleur des cheveux. Cette circonstance a créé un terrain commun de revendication des libertés d'expression pour les deux religions.

- 2007 : au point 26, la Charte des valeurs de la citoyenneté et de l'intégration adoptée par le ministère de l'Intérieur prévoit qu' «en Italie, il n'existe pas de restrictions à la façon de se vêtir pour autant que le choix soit libre, et ne porte pas atteinte à la dignité de la personne. Des formes d'habillement couvrant le visage ne sont pas admissibles parce qu'elles empêchent la reconnaissance de la personne et la gêne au moment d'entrer en contact avec les autres ».

- Juin 2008 : la VI ection du Conseil d'État prononce la décision $\mathrm{n}^{\circ} 3076$, qui met fin au chaos provoqué par les nombreuses ordonnances municipales étendant l'application de la loi 152, avec une interprétation suggestive, à la burqa et au niqab en les considérant comme un masque. En substance, cet arrêt permet de porter n'importe quel vêtement religieux, estimant le rappel à la loi inapplicable, et satisfait les besoins 
de sûreté publique par l'obligation de retrait du voile pour favoriser l'identification.

- 2008/2010 : des enquêtes cognitives et des auditions législatives sont entamées et les premiers projets de loi pour la modification de la loi 152 sont présentés devant les commissions des affaires institutionnelles. Toutefois, le Parlement ne parvient pas à édicter une interdiction explicite puisque tant les législateurs que les experts italiens et étrangers se sont montrés partagés en ce qui concerne le mode de vivre et de traduire le sentiment religieux, ainsi que sur la liaison entre la sûreté publique, la burqa et le niqab, et le fondamentalisme islamique.

Selon la jurisprudence en vigueur, le lieu public est un endroit librement accessible à tous, sans limites particulières (une route, une plage, etc.) ; en revanche, le lieu ouvert au public se caractérise par l'accès libre mais réglementé en quelque sorte par le propriétaire public ou privé (horaires d'ouverture, paiement d'un ticket d'entrée, etc.) ; enfin un lieu exposé au public désigne l'espace, ce qui s'y trouve et ce qui s'y passe, susceptible d'être exposé à la vision d'un public général de personnes (par exemple dans une pièce privée visible par la fenêtre). Il semble donc possible d'affirmer qu'un lieu de culte peut être considéré comme un lieu ouvert au public.

- Début 2011 : premier oui de la commission des Affaires constitutionnelles de la Chambre en faveur de la proposition de loi, qui se propose d'interdire la burqa et le niqab dans les lieux publics parce qu'ils ne permettent pas l'identification du visage d'une personne. La proposition prévoit une modification de l'article 5 de la loi 152, en stipulant qu'il "est défendu de cacher ou de déguiser son visage ou de rendre impossible la reconnaissance personnelle dans un lieu public ou ouvert au public, même moyennant des casques de protection, des vêtements ou des accessoires en tous genres, y compris ceux qui sont d'origine ethnique et culturelle, à l'instar de la burqa et du niqab ». Elle prévoit également des exceptions pour des motifs professionnels ou de santé ou pour la participation à des manifestations sportives, artistiques ou traditionnelles.

- Septembre 2011 : la commission des Affaires constitutionnelles de la Chambre a approuvé à titre définitif le projet de loi qui permet le port de la burqa, avec le visage découvert, dans l'espace public pour " intégrer et respecter les cultures religieuses de tout un chacun sans perdre de vue le besoin de protéger et de garantir la sécurité générale ».

$* * *$

En conclusion, nous pouvons affirmer que l'émancipation de la femme musulmane passe par le port du voile ou par son refus, puisque ce voile peut avoir un sens personnel séculier ou religieux, et symboliser aussi bien la tradition que l'émancipation et la lutte. Nous devons toutefois réellement espérer que les parlements européens s'intéressent aux femmes au-delà du 
symbole que représente la burqa, en leur garantissant la jouissance et la défense des droits à la participation, au fait de ne pas subir de violence et d'être écoutées, ainsi que celles de tous les droits économiques et sociaux sans lesquels la liberté, l'autonomie, la dignité et l'intégration ne sont que des concepts sans consistance.

À toutes les femmes libres dans l'âme mais non dans leur corps. 